Provided for non-commercial research and education use. Not for reproduction, distribution or commercial use.

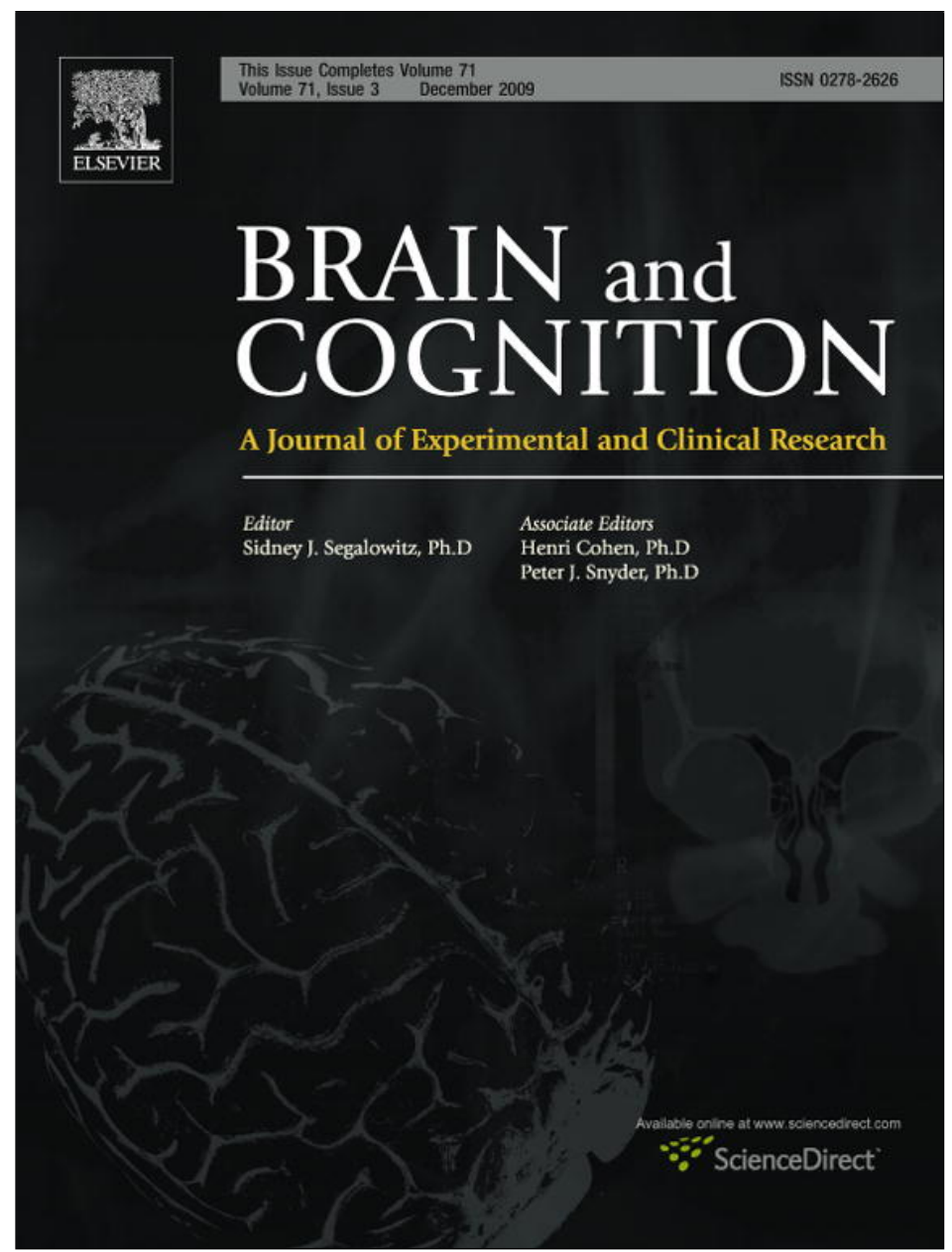

This article appeared in a journal published by Elsevier. The attached copy is furnished to the author for internal non-commercial research and education use, including for instruction at the authors institution and sharing with colleagues.

Other uses, including reproduction and distribution, or selling or licensing copies, or posting to personal, institutional or third party websites are prohibited.

In most cases authors are permitted to post their version of the article (e.g. in Word or Tex form) to their personal website or institutional repository. Authors requiring further information regarding Elsevier's archiving and manuscript policies are encouraged to visit:

http://www.elsevier.com/copyright 


\title{
Association of catechol-O-methyltransferase (COMT) polymorphism and academic achievement in a Chinese cohort
}

\author{
Ting-Kuang Yeh ${ }^{\mathrm{a}, \mathrm{b}, \mathrm{c}}$, Chun-Yen Chang ${ }^{\mathrm{a}, \mathrm{c}, *}$, Chung-Yi Hu${ }^{\mathrm{d}}$, Ting-Chi Yeh ${ }^{\mathrm{e}}$, Ming-Yeh Lin $^{\mathrm{c}, \mathrm{d}}$ \\ ${ }^{a}$ Science Education Center and Graduate Institute of Science Education, National Taiwan Normal University, Taiwan, ROC \\ ${ }^{\mathrm{b}}$ Healthy Center, National Taiwan University, Taiwan, ROC \\ ${ }^{\mathrm{c}}$ Department of Earth Sciences, National Taiwan Normal University, Taiwan, ROC \\ ${ }^{\mathrm{d}}$ Department of Clinical Laboratory Sciences and Medical Biotechnology, College of Medicine, National Taiwan University, Taiwan, ROC \\ ${ }^{\mathrm{e}}$ Department of Pediatric, Mackay Memorial Hospital, Taiwan, ROC
}

\section{A R T I C L E I N F O}

\section{Article history:}

Accepted 26 July 2009

Available online 22 August 2009

\section{Keywords:}

Academic performance

Basic Competency Test

Catechol-O-methyltransferase

Cognition

\begin{abstract}
A B S T R A C T
Catechol-O-methyltransferase (COMT) is a methylation enzyme that catalyzes the degradation pathway and inactivation of dopamine. It is accepted widely as being involved in the modulation of dopaminergic physiology and prefrontal cortex (PFC) function. The COMT Val158Met polymorphism is associated with variation in COMT activity. COMT 158Met allele may be advantageous for PFC-related cognitive abilities; however, it is also associated with increased anxiety, depression, and emotional vulnerability in response to stress or educational adversity. We hypothesized that the COMT polymorphism might be associated with academic performance. In this study, 779 Taiwanese tenth-grade volunteers were recruited. Scores from the Basic Competency Test (BCT), an annual national competitive entrance examination, were used to evaluate academic performance. The results indicated that students bearing homozygous for the Met allele tended to perform more poorly in all BCT subtests as compared to the other groups. In particular, the former performed significantly more poorly in the science and social science subtests. These findings provide evidence that affective factors might overwhelm cognitive abilities in high-stake tests like the BCT.
\end{abstract}

(c) 2009 Elsevier Inc. All rights reserved.

\section{Introduction}

The academic achievement of students has long been regarded as the crucial issue for educational practice. Recent empirical research on education has demonstrated convincingly the associations that exist between academic achievement and cognitive abilities and environmental factors (Rindermann, 2007). Furthermore, after the high heritability of behavior and cognitive abilities was demonstrated by twin and adoption studies, contemporary researchers in the fields of education, psychological science, and neuroscience have all acknowledged the crucial role of genetic factors in learning (Lykken, Bouchard, McGue, \& Tellegen, 1990). However, the nature of the direct link between genes, brain development, and behavior can be difficult to establish. This preliminary study focuses on exploring the association between the dopaminergic system, as assessed through molecular genetic techniques, and academic achievement.

\footnotetext{
* Corresponding author. Address: Science Education Center, National Taiwan Normal University, 88 Sec. 4, Ting-Chou Rd., Taipei 116, Taiwan. Fax: +88622933 3315.

E-mail address: changcy@ntnu.edu.tw (C.-Y. Chang).
}

Dopamine is postulated to be the key neurotransmitter that regulates the function of the prefrontal cortex (PFC), and its role in mediating human cognitive abilities and affective behavioral processes has been accepted widely (Blair, Gamson, Thorne, \& Baker, 2005; Nieoullon, 2002; Previc, 1999; Tunbridge, Harrison, \& Weinberger, 2006). For example, with respect to cognitive orientation, a number of studies have indicated that a deficit in dopamine levels in the brain might be associated with impaired cognitive performance in the Wisconsin Card Sorting Test and Tower of London Test (Nieoullon, 2002). Volkow et al. (1998) have reported that decreases in brain dopamine activity may contribute to impaired performance in tasks that involve prefrontal brain regions, and Previc (1999) have indicated that increased dopamine levels in the brain can contribute to an increase in analytical intelligence. With respect to affective orientation, as reviewed by Tunbridge et al. (2006), changes in dopamine levels in the brain are associated with a variety of psychiatric symptoms. For example, increased dopamine activity has been reported to be associated with productive positive symptoms of psychosis in schizophrenia, whereas, in contrast, Parkinson's disease is associated with a reduced dopamine concentration in the brain. Furthermore, the tonic/phasic dopamine theory suggests that phasic dopamine may be important for updating or resetting working memory traces, principally via 
D2 dopamine receptors, whereas tonic dopamine may enhance the stability of these traces, principally via D1 receptors (Grace, 1991; Nolan, Bilder, Lachman, \& Volavka, 2004). Numerous evidence in the literature shows that increasing tonic dopamine may promote cognitive stability but limit cognitive flexibility (Bilder, Volavka, Lachman, \& Grace, 2004; Drabant et al., 2006; Nolan et al., 2004).

Catechol-O-methyltransferase (COMT) is a methylation enzyme that catalyzes the first step of the dopamine degradation pathway and thus inactivates the neurotransmitter. It is the key regulator with respect to the modulation of dopamine levels, cognitive functioning, and neural activity of the PFC. The COMT gene (Gene ID: 1312) is located on chromosome 22q11.21 and consists of six exons. A single nucleotide polymorphism (SNP), which corresponds to a $G$ to $A$ transition at nucleotide 472 in exon 4 of the COMT gene and results in a valine to methionine substitution at residue 158 , is associated with differential COMT activity (Syvanen, Tilgmann, Rinne, \& Ulmanen, 1997). The 158Met allele of the COMT gene, which decreases the thermostability and activity of the enzyme and thereby reduces the rate of dopamine catabolism, is associated with higher dopamine concentrations in the PFC than is the 158Val polymorphism (Lachman et al., 1996; Palmatier, Kang, \& Kidd, 1999).

A number of studies have shown that healthy individuals that bear the COMT 158Met/Met genotype exhibit superior cognitive performance in the Wisconsin Card Sorting Test in comparison with those that carry the 158Val/Val genotype (Bruder et al., 2005; Malhotra et al., 2002). Egan et al. (2001) have indicated that, in schizophrenia patients, the 158 Met variant is associated with enhanced performance in a working memory task. Barnett et al. (2007) have reported that the COMT 158Met allele is associated with better performance in terms of verbal IQ and executive function scores in adolescent boys. Taken together, these data suggest that the COMT 158Met/Met genotype is advantageous for PFC function and PFC-related cognitive abilities. However, it has been reported widely that the $158 \mathrm{Met}$ allele may be associated with negative affective disorders. For example, a number of studies have proposed the association of the COMT 158Met allele with increased anxiety, major depression, bipolar affective disorder, and increased inflexible behavior in response to negative emotional stimuli (Bishop, Fossella, Croucher, \& Duncan, 2008; Drabant et al., 2006; Enoch, Waheed, Harris, Albaugh, \& Goldman, 2009; Smolka et al., 2005; Tunbridge et al., 2006; Zubieta et al., 2003). In addition, neural imaging studies revealed that increased anxiety and emotional vulnerability in association with COMT 158Met allele may result from changes in the limbic system and hippocampus activation (Drabant et al., 2006; Smolka et al., 2005). As Enoch et al. (2009) have illustrated, although carriers of the 158Met allele show enhanced PFC-related cognitive abilities, when they are exposed to stress or educational adversity, their academic ability may be overwhelmed by their emotional vulnerability. 158Val homozygotes may be more resilient to educational adversity.

In Taiwan, students who wish to attend secondary level education, after junior high school, must take the annual Basic Competency Test (BCT), a national standardized test that measures educational achievement, in order to enroll in a senior high school. In general, more than 310,000 examinees (equal to $95 \%$ of this age group) take the BCT each year. At present, it is estimated that only $39 \%$ of the age group is allowed to proceed to senior high school (Chang \& Cheng, 2008). This type of competitive selection process has survived for many years in traditional Asian culture because it provides an opportunity for low-ranking socioeconomic groups to move upwards in society. However, in this competitive environment, the students are subjected to extreme academic pressure as a result of the intense long-term preparation that is required and the implications for their future education.
We hypothesize that the COMT Val158Met polymorphism is associated with the academic performance of students in the BCT. To the best of our knowledge, few studies have investigated the correlation between genotype and academic performance. This study attempted to redress the balance. It is our hope that this research will clarify future directions for the investigation of behavioral mechanisms for researchers in various fields.

\section{Materials and methods}

\subsection{Academic achievement scores}

The BCT consists of a 2-day written test program that covers six subjects: Chinese, English, mathematics, science, social science, and writing. Subtests within the BCT consist of 34-63 multiple choice questions, which include testlets and simple items. Examinees have $70 \mathrm{~min}$ to complete each subtest. Table 1 shows examples of questions from the BCT subtests. Item response theory (IRT) models (Bock, Thissen, \& Zimowski, 1997; Lord, 1980) are used to convert the examinees' raw scores to a score scale (for the writing test the range is $0-10$; for the other subtests the range is $0-60$ ). This enables information on score precision to be incorporated into the score scale and facilitates correct interpretation of the scores. The BCT scores are published and examinees are assigned to the national senior high schools on the basis of their total BCT scores. This screening process streams students with similar total BCT scores into the same senior high school.

\subsection{Participants}

In order to select a nationally-representative sample in terms of academic achievement, four senior high schools were selected. In the first school (located in southern Taiwan) the pupils obtained high BCT scores, in the second (also located in southern Taiwan) the pupils obtained low BCT scores, and in the remaining two (one located in the middle of and the other in northern Taiwan), they obtained medium BCT scores. A total of 779 tenthgrade volunteers, 314 of whom were male and all of whom were Han Chinese, were recruited for this study. The mean age of the subjects was 16.3 years (SD, 0.5 ; age range: $16-17$ years). Statistical analysis of goodness-of-fit showed that when the scores of these senior high school students were combined, they gave a good representation (simulation) of the national probability distribution $\left(\chi^{2}=21\right)$. All the volunteers underwent clinical/biochemical assessments, which included the measurement of blood pressure, heart rate, hemoglobin, uric acid, creatinine, alanine transaminase, and aspartate transaminase, and psychological interview by a physician. All the volunteers were found to be physically and psychologically healthy. The volunteers and their parents were all informed explicitly about the plan, protocol, and procedure for the study and written consent was obtained prior to the study being performed. This study was approved by the institutional review board of the National Taiwan University Hospital and the ClinicalTrials.gov registry of clinical trials (ClinicalTrials.gov identifier: NCT00713570).

\subsection{Genotyping}

We designed primers to amplify the genomic sequence that contained the Val158Met polymorphism (rs4680) in exon 4 of the COMT gene (GeneID: 1312): $5^{\prime}$-ACG CCG TGA TTC AGG AGC A-3' (COMT-s) and 5'-CGT CTT TCC TCA GCC CCA G-3' (COMT-as). Amplification was carried out using recombinant TaKaRa Taq ${ }^{\text {TM }}$ DNA Polymerase (Takara Bio Inc., Tokyo, Japan) and a PX2 Thermal Cycler (Thermo Electron Corporation, USA). The conditions for the 
Table 1

Examples of questions from the different BCT subtests.

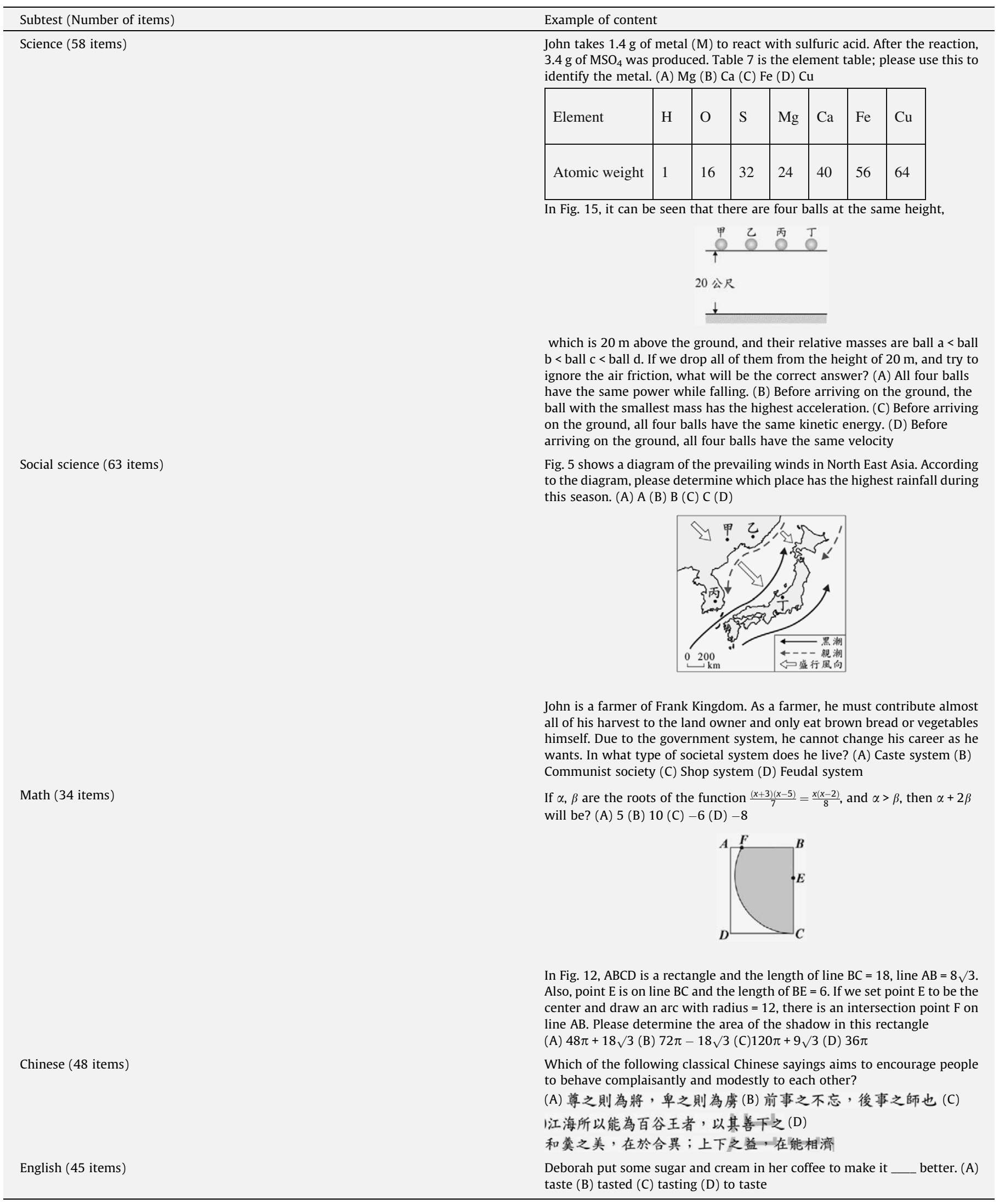

PCR consisted of an initial incubation at $95^{\circ} \mathrm{C}$ for $1 \mathrm{~min}$, followed by 35 cycles of $95^{\circ} \mathrm{C}$ for $1 \mathrm{~min}, 55^{\circ} \mathrm{C}$ for $30 \mathrm{~s}$, and $72^{\circ} \mathrm{C}$ for $30 \mathrm{~s}$.
The PCR products (350 bp) were visualized on a $1.5 \%$ agarose gel stained with ethidium bromide. 
Table 2

The COMT-158 genotype-specific probes designed for SSOPH typing.

\begin{tabular}{lll}
\hline Name & Sequence & Assay conditions \\
\hline COMTE4-all & $5^{\prime}$-Biotin- & $5 \times 42^{\circ} \mathrm{C}, 10 \mathrm{~min} /$ \\
& CATCGAGATCAACCCC & $0.1 \times 42^{\circ} \mathrm{C}$, \\
& & $10 \mathrm{~min}$ \\
COMTE4- & $5^{\prime}$-Biotin- & $2 \times 42^{\circ} \mathrm{C}, 10 \mathrm{~min} /$ \\
158(G) & CGCTGGCGTGAAGGA & $0.1 \times 42^{\circ} \mathrm{C}$, \\
& & $10 \mathrm{~min}$ \\
COMTE4- & $5^{\prime}$-Biotin- & $5 \times 42^{\circ} \mathrm{C}, 15 \mathrm{~min} /$ \\
158(A) & CGCTGGCATGAAGAA & $0.2 \times 42^{\circ} \mathrm{C}$, \\
& & $10 \mathrm{~min}$ \\
\hline
\end{tabular}

A pilot study was performed using DNA samples from 20 healthy subjects. The PCR products obtained from these subjects, as described above, were sequenced using an ABI Prism ${ }^{\circledR}$ BigDye $^{\mathrm{TM}}$ Terminator v3.1 Ready Reaction Cycle Sequencing kit and an ABI Prism $^{\circledR} 3730$ Genetic Analyzer (Applied Biosystems, Foster City, CA, USA). This allowed the identification of PCR products that corresponded to the COMT $158 \mathrm{GG}, \mathrm{AG}$, and AA genotypes, and these were utilized as positive controls for the following genotyping procedures.

The genetic polymorphism at residue 158 of COMT gene was analyzed using the PCR-based sequence-specific oligonucleotide probe hybridization (PCR-SSOPH) method. Eight microliters of the PCR products were denatured with $50 \mu \mathrm{l}$ of $0.4 \mathrm{~N} \mathrm{NaOH} /$ $25 \mathrm{mM}$ EDTA for $10 \mathrm{~min}$, and then dot-blotted onto a nylon membrane (Biodyne B Membrane; Pall, Glen Cove, NY, USA) in an $8 \times 12$ format. The PCR products that corresponded to the COMT $158 \mathrm{GG}$, AG and AA alleles, as confirmed by sequencing, were included on each blot as controls. The membranes were then hybridized with 5 '-biotinylated SSO probes (Table 2) in the presence of streptavidin-conjugated horseradish peroxidase (Roche, Basel, USA), and subjected to stringent washing to allow identification of the genotype. Hybridization was visualized by chemiluminescence using the ECL Gene Detection System (GE HealthcareLife Science, Uppsala, Sweden).

\subsection{Statistical analysis}

In this study, the subjects were grouped according to their COMT 158 genotype (AA, AG or GG, which encodes Val/Val, Val/ Met or Met/Met at residue 158 of COMT, respectively), and the $\chi^{2}$ test was used to test for Hardy-Weinberg equilibrium. Univariate ANOVA was used to determine separately the statistical significance of cognitive ability among the three genotype groups. The level of confidence was set at the 0.05 significance level. Post-hoc testing was performed using Scheffe's $F$ test because this has been demonstrated to be the most powerful post-hoc multiple comparison procedure (Klockars \& Hancock, 2000). To meet contemporary calls for improvement in the interpretation and reporting of quantitative research in education (Rennie, 1998; Thompson, 1996), we have reported practical significance (effect magnitudes) along with each statistical significance test. The effect size index $f$ was used, because it is more appropriate for the analysis of variance or covariance (Cohen, 1988). According to Cohen's rough characterization, $f=0.1$ is deemed to be a small effect size, $f=0.25$ a medium effect size, and $f=0.4$ a large effect size. Tests of the assumptions used for ANOVA and inferential statistical analyses were performed using SPSS ver. 13.0 (Chicago, IL, USA).

\section{Results}

The genotypic distributions of the subjects are presented in Table 3 . Of the 779 healthy subjects, $60(8 \%)$ were homozygous for the COMT 158Met allele, 388 (50\%) were homozygous for COMT $158 \mathrm{Val}$, and 331 (42\%) were COMT 158Val/Met heterozygotes. The observed distribution of genotypes was consistent with that expected for Hardy-Weinberg equilibrium $\left(\chi^{2}=0.853, p>0.05\right)$, which confirmed that no genotype was being sampled selectively. Furthermore, the genotype frequencies in our population were consistent with the HapMap-HCB population studies reported in the International HapMap project (http://www.hapmap.org). The three genotype groups did not show significant differences with respect to gender $\left(\chi^{2}=1.5, p>0.05\right)$, age $\left(\chi^{2}=0.38, p>0.05\right)$ or school $\left(\chi^{2}=3.74, p>0.05\right)$.

The academic achievement (BCT score) of the students in the different COMT genotype groups was analyzed by ANOVA (as shown in Table 3). The major finding of this study was that COMT 158Met/Met homozygous students tended to perform relatively poorly in all BCT subtests as illustrated in Fig. 1. The association between the COMT genotypes and each individual academic subtest was investigated further. No significant differences among the three genotype groups were found for the Chinese, English, or writing scores. However, significant differences were identified among the three groups with respect to the science ( $F$ $(2,776)=4.75, p<0.01, \eta^{2}=0.018, f=0.14$, small to medium effect size) and social science scores $(F(2,776)=4.08, p=0.02$, $\eta^{2}=0.011, f=0.11$, small effect size). For mathematics, marginally significant differences were identified among the three genotype groups $\left(F(2,776)=2.87, p=0.06, \eta^{2}=0.09, f=0.1\right.$, small effect size). Post-hoc Scheffe analysis revealed that the Met/Met group performed significantly poorer in the science and social science subtests as compared with the Val/Met and Val/Val groups, as shown in Table 3. In summary, the COMT Val158Met polymorphism is associated with differences in performance in the science and social science subtests of the BCT, and therefore, may be implicated in different learning capabilities in science and social science subjects.

Table 3

BCT scores in students with different COMT7-158 genotypes.

\begin{tabular}{|c|c|c|c|c|c|c|}
\hline \multirow[t]{2}{*}{ COMT residue 158} & \multicolumn{3}{|c|}{ COMT-158 genotype } & \multirow{2}{*}{$\begin{array}{l}F \\
\text { ANOVA }(p)\end{array}$} & \multirow[t]{2}{*}{$f$} & \multirow{2}{*}{$\begin{array}{l}\text { Post-hoc } \\
\text { Scheffe }(p)\end{array}$} \\
\hline & Met/Met $(n=60)$ & Met/Val $(n=331)$ & Val/Val $(n=388)$ & & & \\
\hline \multicolumn{7}{|l|}{ Test subjects } \\
\hline Science & $39.4(9.5)^{\mathrm{a}}$ & $41.9(9.1)$ & $43.1(8.5)$ & $4.75(<\mathbf{0 . 0 1})$ & 0.14 & $\mathrm{MM}<\mathrm{VV}^{* *}$ \\
\hline Social science & $41.3(9.3)$ & $44.2(8.3)$ & $44.5(7.8)$ & $4.08(\mathbf{0 . 0 2})$ & 0.11 & $\begin{array}{l}\mathrm{MM}<\mathrm{VV}^{* *} \\
M M<M V^{*}\end{array}$ \\
\hline Mathematics & $42.1(9.0)$ & $43.6(8.4)$ & $44.6(7.9)$ & $2.87(0.06)$ & 0.10 & \\
\hline English & $47.2(9.2)$ & $49.9(9.0)$ & $49.5(9.2)$ & $1.47(0.23)$ & 0.06 & \\
\hline Chinese & $40.9(9.6)$ & $43.5(8.5)$ & $43.3(8.6)$ & $2.31(0.10)$ & 0.08 & \\
\hline Writing & $8.53(1.5)$ & $8.57(1.3)$ & $8.54(1.2)$ & $0.06(0.94)$ & 0.01 & \\
\hline
\end{tabular}

${ }^{a}$ Mean (SD).

$p<0.05$.

$p<0.01$. 


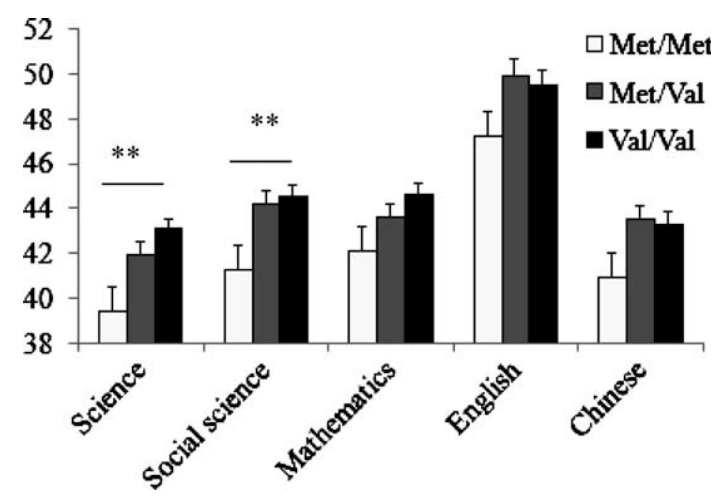

Fig. 1. Comparison of BCT subject scores among genotypes.

\section{Discussion}

The major finding of this study was that COMT 158Met/Met homozygous students showed a trend towards poorer performance in all subtests of the BCT as compared to students homozygous for $\mathrm{Val} /$ Val or heterozygous for Val/Met. In particular, the performance of the COMT 158Met/Met homozygous students in the science and social science subtests was significantly poorer than that of the other two groups (Fig. 1).

As mentioned above, the annual BCT in Taiwan is a high-stake test that is used to determine which students can enter into senior high school and which school they will be assigned to. Therefore, students are subjected to intense pressure due to the competition and long-term preparation for this examination, and the impact that it will have on their future educational opportunities. Previous studies have indicated that the COMT Val158Met polymorphism plays a key role in PFC-related cognitive abilities and affective behavioral processes. Carriers of the Met allele may benefit from enhanced working memory and verbal memory. However, the Met allele may also be associated with increased anxiety, major depression, and emotional instability and vulnerability when the carrier is exposed to stress or educational adversity. At the educational level, a number of studies have reported that affective factors, such as testing and learning anxiety, the negative impact of emotional vulnerability, and academic stress, can have a major influence on academic performance (Hembree, 1988; Ng, Koh, \& Chia, 2003; Zajacova, Lynch, \& Espenshade, 2005). The results of the current study suggest that the tendency of COMT 158Met/ Met homozygous students to perform relatively poorly in all subtests of the BCT might result from the predominant role of stress and emotional vulnerability.

The significant effect of COMT genotype on the performance of students in the science and social science subtests of the BCT might be due to the increased stress and anxiety that is associated with these subjects. In recent years, curriculum and examination reform has received much attention from teachers and researchers in science education around the world; Taiwan is no exception. With respect to national entrance examinations in Taiwan, such as the BCT and college entrance examinations, the importance of evaluating the application, reasoning, and problem-solving abilities of students, rather than merely testing knowledge memorized by rote, has been highlighted (Chang \& Cheng, 2008). This is especially the case for socalled "applied science" (which includes science and social science subjects). In applied science examinations, students not only have to memorize substantial amounts of information but also have to apply this knowledge to solving integrated problems. The need to utilize "higher order" cognitive abilities, such as problem solving, classification, reasoning, and critical thinking abilities, when preparing academic subjects may increase the cognitive load of the students and result in higher levels of stress. It has long been suggested that students suffer higher levels of academic anxiety in science and mathematics subjects as compared to subjects that are based on memorization, language or assembling knowledge from experience (Everson, Tobias, Hartman, \& Gourgey, 1993; Hembree, 1988). Therefore, the significantly poorer performance of the COMT $158 \mathrm{Met} / \mathrm{Met}$ homozygous students in the science and social science subtests as compared to the language-based subjects may be due to the different levels of stress and emotional loading that are induced by different academic subjects.

Recent reports have shown that the Met allele, which results in increased levels of tonic dopamine release but decreased levels of phasic dopamine release, may be associated with increased cognitive stability and decreased cognitive flexibility during neural-network activation (Bilder et al., 2004; Drabant et al., 2006; Nolan et al., 2004). In addition, Bishop et al. (2008) have used functional magnetic resonance imaging to demonstrate that the COMT 158Val allele is associated with enhanced fluid intelligence, which includes problem solving, classification, reasoning, and critical thinking. In contrast, carriers of the COMT 158Met allele might perform better in terms of executive function, working memory, and cognitive stability but more poorly with respect to aspects of fluid intelligence. As mentioned previously, the BCT has focused recently more on evaluating the application, reasoning, and problem-solving abilities of students, rather than testing knowledge memorized by rote (Chang \& Cheng, 2008). Therefore, it is possible that the performance of students in the BCT, especially in the science and social science subtests, might be influenced substantially by the beneficial cognitive effects of the $158 \mathrm{Val}$ allele. However, due to the lack of measurement of the emotional effects of the BCT and of cognitive abilities at the experimental or meta-level, the current study cannot establish a connection between these two factors. In subsequent studies, it would be of interest to perform rigorous hypothesis testing regarding this issue by recording and analyzing more complete information concerning the students' cognitive abilities and behavior, together with environmental information.

Educational/cognitive researchers might be interested in the implications of the COMT Val158Met polymorphism. During the learning process, students who do not perform as well academically as others due to poor cognitive abilities or emotional self-control show a decreased willingness to learn. Therefore, the genotyping of students could give strategic educationists an understanding of the innate cognitive abilities and emotional self-control of an individual student. They could then give priority to subjects that match the strongest cognitive ability of the student, and monitor the emotions of the student in relation to learning. In this way, the student's interest in learning and achievement could be increased.

This study has a number of limitations. As mentioned previously, researchers in cognition and education acknowledge the high degree of complexity that underlies cognitive behavior. It is reasonable to infer that the academic achievement of students might be affected by the interaction of multiple factors, such as multiple genes, various environmental factors, and epigenetic regulation. Furthermore, it is noteworthy that the students in this study were physically and psychologically healthy. Consequently, it will be more difficult to attain statistical/practical significance with respect to cognitive performance among different COMT genotype groups in a healthy population than in patients that are suffering from single-gene diseases or psychiatric disorders. In a healthy population, it is more likely that results of marginally practical significance will be obtained. However, statistically significant results with small to medium effect sizes might be useful for researchers in cognitive psychology and neuroscience, and assist in their further exploration of behavioral mechanisms on the cognitive/neural/molecular levels.

Many studies across a range of different social cultures, for example the United States, United Kingdom and Asia, have re- 
ported that a large proportion of students experience higher levels of examination stress than those from other countries (Crystal et al., 1994; Denscombe, 2000). Therefore, it is reasonable to infer that the correlation between the COMT 158Met allele and higher academic stress/lower academic performance may be a fundamental characteristic of the adolescent population in many countries. However, it is interesting and noteworthy that the frequency of the COMT 158Met allele within Asian populations is significantly lower than that in Caucasian populations (Palmatier et al., 1999). Therefore, we acknowledge that future studies in different groups, such as in students or patients of different ethnic origins, are especially important to validate further the effect of the COMT 158 genetic polymorphism on learning and cognition.

In addition, the academic achievement of students might be affected by the interaction of multiple factors, such as multiple genes, various environmental factors, and epigenetic regulation. We have only examined the effects of a single genetic polymorphism. Further analyze with other possible candidate genes in dopaminergic system would greatly help researchers to discriminate the genotypic effects. Besides, socioeconomic background might play an important and potential role in students' academic performance. For example, students with better socioeconomic background might receive more opportunities in informal education. We thought it would be interesting to analyze the impact of interaction between COMT genotypes and socioeconomic factor $(\mathrm{G} \times \mathrm{E})$ in students' academic performance. With regards to furthering this study, additional investigations using an enlarged sample size to give more reliable data and clinical trials to evaluate the effects of COMT polymorphisms on cognitive ability are underway. We envisage that the integrated and progressive study of human learning behavior will provide researchers in different fields with concrete evidence that can be used to untangle the complicated relationships/mechanisms of behavior.

\section{Acknowledgments}

The work in this study was supported by the National Science Council of Taiwan under contracts NSC 96-2511-S-003-022-MY2 and NSC 98-2511-S-003-050-MY3. The authors gratefully acknowledge the assistance provided by Sheng-Kai Chang for management of COMT genotyping; Hsueh-Jen Tsao, Ssu-Lang Lin, TseMin Wu, Hsueh-Kuei Hu, Chien-Hua Hsiao, Fu-Tai Chuang, KuanHong Lin, Chen-Tsung Cheng, Jih Jung Hsieh, Chang-Pu Liu for their consulting for recruitment of participants. Above all, we appreciated all the students and their families for giving their consent to participate in this project. The authors gratefully thank the editor and anonymous reviewer for their insightful comments on an earlier version of the manuscript.

\section{References}

Barnett, J. H., Heron, J., Ring, S. M., Golding, J., Goldman, D., Xu, K., et al. (2007). Gender-specific effects of the catechol-O-methyltransferase $\operatorname{Val}(108) /(158) \mathrm{Met}$ polymorphism on cognitive function in children. American Journal of Psychiatry, $164,142-149$.

Bilder, R. M., Volavka, J., Lachman, H. M., \& Grace, A. A. (2004). The catechol-Omethyltransferase polymorphism: Relations to the tonic-phasic dopamine hypothesis and neuropsychiatric phenotypes. Neuropsychopharmacology, 29, 1943-1961.

Bishop, S. J., Fossella, J., Croucher, C. J., \& Duncan, J. (2008). COMT val(158)met genotype affects recruitment of neural mechanisms supporting fluid intelligence. Cerebral Cortex, 18, 2132-2140.

Blair, C., Gamson, D., Thorne, S., \& Baker, D. (2005). Rising mean IQ: Cognitive demand of mathematics education for young children, population exposure to formal schooling, and the neurobiology of the prefrontal cortex. Intelligence, 33, 93-106.

Bock, R. D., Thissen, D., \& Zimowski, M. F. (1997). IRT estimation of domain scores. Journal of Educational Measurement, 34, 197-211.

Bruder, G. E., Keilp, J. G., Xu, H. Y., Shikhman, M., Schori, E., Gorman, J. M., et al. (2005). Catechol-O-methyltransferase (COMT) genotypes and working memory:
Associations with differing cognitive operations. Biological Psychiatry, 58, 901-907.

Chang, C. Y., \& Cheng, W. Y. (2008). Science achievement and students' selfconfidence and interest in science: A Taiwanese representative sample study. International Journal of Science Education, 30, 1183-1200.

Cohen, J. (1988). Statistical power analysis for the behavioral sciences (2nd ed.). Hillsdale, NJ: Erlbaum.

Crystal, D. S., Chen, C. S., Fuligni, A. J., Stevenson, H. W., Hsu, C. C., Ko, H. J., et al. (1994). Psychological maladjustment and academic achievement: A crosscultural study of Japanese, Chinese, and American high school students. Child Development, 65, 738-753.

Denscombe, M. (2000). Social conditions for stress: Young people's experience of doing GCSEs. British Educational Research Journal, 26, 359-374.

Drabant, E. M., Hariri, A. R., Meyer-Lindenberg, A., Munoz, K. E., Mattay, V. S., Kolachana, B. S., et al. (2006). Catechol O-methyltransferase val(158)met genotype and neural mechanisms related to affective arousal and regulation. Archives of General Psychiatry, 63, 1396-1406.

Egan, M. F., Goldberg, T. E., Kolachana, B. S., Callicott, J. H., Mazzanti, C. M., Straub, R. E., et al. (2001). Effect of COMT Val(108/158) Met genotype on frontal lobe function and risk for schizophrenia. Proceedings of the National Academy of Sciences of the United States of America, 98, 6917-6922.

Enoch, M.-A., Waheed, J. F., Harris, C. R., Albaugh, B., \& Goldman, D. (2009). COMT Val158Met and cognition: Main effects and interaction with educational attainment. Genes, Brain and Behavior, 8, 36-42.

Everson, H. T., Tobias, S., Hartman, H., \& Gourgey, A. (1993). Test anxiety and the curriculum: The subject matters. Anxiety, Stress, and Coping, 6, 1-8.

Grace, A. A. (1991). Phasic versus tonic dopamine release and the modulation of dopamine system responsivity: A hypothesis for the etiology of schizophrenia. Neuroscience, 41, 1-24.

Hembree, R. (1988). Correlates, causes, effects, and treatment of test anxiety. Review of Educational Research, 58, 47-77.

Klockars, A. J., \& Hancock, G. R. (2000). Scheffe's more powerful F-protected post hoc procedure. Journal of Educational and Behavioral Statistics, 25, 13-19.

Lachman, H. M., Papolos, D. F., Saito, T., Yu, Y. M., Szumlanski, C. L., \& Weinshilboum, R. M. (1996). Human catechol-O-methyltransferase pharmacogenetics: Description of a functional polymorphism and its potential application to neuropsychiatric disorders. Pharmacogenetics, 6, 243-250.

Lord, E. M. (1980). Applications of item response theory to practical testing problems. Hillsdale, NJ: Erlbaum.

Lykken, D. T., Bouchard, T. J., McGue, M., \& Tellegen, A. (1990). The Minnesota twin family registry: Some initial findings. Acta Geneticae Medicae et Gemellologiae, 39, 35-70.

Malhotra, A. K., Kestler, L. J., Mazzanti, C., Bates, J. A., Goldberg, T., \& Goldman, D. (2002). A functional polymorphism in the COMT gene and performance on a test of prefrontal cognition. American Journal of Psychiatry, 159, 652-654.

$\mathrm{Ng}$, V., Koh, D., \& Chia, S. E. (2003). Examination stress, salivary cortisol, and academic performance. Psychological Reports, 93, 1133-1134.

Nieoullon, A. (2002). Dopamine and the regulation of cognition and attention. Progress in Neurobiology, 67, 53-83.

Nolan, K. A., Bilder, R. M., Lachman, H. M., \& Volavka, J. (2004). Catechol Omethyltransferase $\operatorname{Val}(158)$ Met polymorphism in schizophrenia: Differential effects of Val and Met alleles on cognitive stability and flexibility. American Journal of Psychiatry, 161, 359-361.

Palmatier, M. A., Kang, A. M., \& Kidd, K. K. (1999). Global variation in the frequencies of functionally different catechol-O-methyltransferase alleles. Biological Psychiatry, 46, 557-567.

Previc, F. H. (1999). Dopamine and the origins of human intelligence. Brain and Cognition, 41, 299-350.

Rennie, L. J. (1998). Improving the interpretation and reporting of quantitative research. Journal of Research in Science Teaching, 35, 237-248.

Rindermann, H. (2007). The g-factor of international cognitive ability comparisons: The homogeneity of results in PISA, TIMSS, PIRLS and IQ-tests across nations. European Journal of Personality, 21, 667-706.

Smolka, M. N., Schumann, G., Wrase, J., Grusser, S. M., Flor, H., Mann, K., et al. (2005). Catechol-O-methyltransferase val(158)met genotype affects processing of emotional stimuli in the amygdala and prefrontal cortex. Journal of Neuroscience, 25, 836-842.

Syvanen, A. C., Tilgmann, C., Rinne, J., \& Ulmanen, I. (1997). Genetic polymorphism of catechol-O-methyltransferase (COMT): Correlation of genotype with individual variation of S-COMT activity and comparison of the allele frequencies in the normal population and Parkinsonian patients in Finland. Pharmacogenetics, 7, 65-71.

Thompson, B. (1996). AERA editorial policies regarding statistical significance testing: Three suggested reforms. Educational Researcher, 25, 26-30.

Tunbridge, E. M., Harrison, P. J., \& Weinberger, D. R. (2006). Catechol-omethyltransferase, cognition, and psychosis: Val158Met and beyond. Biological Psychiatry, 60, 141-151.

Volkow, N. D., Gur, R. C., Wang, G. J., Fowler, J. S., Moberg, P. J., Ding, Y. S., et al. (1998). Association between decline in brain dopamine activity with age and cognitive and motor impairment in healthy individuals. The American Journal of Psychiatry, 155, 344-349.

Zajacova, A., Lynch, S. M., \& Espenshade, T. J. (2005). Self-efficacy, stress, and academic success in college. Research in Higher Education, 46, 677-706.

Zubieta, J. K., Heitzeg, M. M., Smith, Y. R., Bueller, J. A., Xu, K., Xu, Y. J., et al. (2003). COMT val(158)met genotype affects mu-opioid neurotransmitter responses to a pain stressor. Science, 299, 1240-1243. 